\title{
The Efficacy and Safety of Endoscopic Sleeve Gastroplasty as an Alternative to Laparoscopic Sleeve Gastrectomy
}

\author{
Jin Young Yoon ${ }^{1}$, Román Turró Arau' ${ }^{2}$, and The Study Group for Endoscopic Bariatric and Metabolic Therapies of the Korean \\ Society of Gastrointestinal Endoscopy \\ ${ }^{1}$ Division of Gastroenterology, Department of Internal Medicine, Kyung Hee University Hospital, Seoul, Korea, ${ }^{2}$ Department of Bariatric \\ Endoscopy, Centro Medico Teknon, Barcelona, Spain
}

Endoscopic sleeve gastroplasty (ESG) is a therapeutic endoscopic technique for reducing the size of the gastric reservoir in obese patients, using a full-thickness endoscopic suturing device. The effectiveness of ESG in weight loss is significantly greater than that of high-intensity diet and lifestyle therapy and lower than that of laparoscopic sleeve gastrectomy (LSG). The efficacy at 12 months after ESG in terms of percentage of total body weight loss and excess body weight loss was approximately $16 \%$ and $60 \%$, respectively. The well-known predictive factors for increased weight loss by ESG are good compliance with regular monitoring and post-procedure care involving a multidisciplinary team approach. Although the underlying mechanism of weight loss induced by ESG is debatable, delayed gastric emptying and early satiation are some of the proposed mechanisms. The pooled rate of adverse events after ESG reported in several meta-analysis studies ranged from $1.5 \%$ to $2.3 \%$ and the incidence of new-onset gastroesophageal reflux disease after ESG was negligible, indicating that ESG has a superior safety profile to LSG. Moreover, ESG reduced the risk of obesity-related metabolic comorbidities, evidenced by the reduction in HbAlc level, systolic blood pressure, triglyceride level, and risk of hepatic steatosis and fibrosis; it even improved the quality of life. ESG could be considered safe and qualify as an alternative treatment to LSG. Clin Endosc 2021;54:17-24

Key Words: Efficacy; Endoscopic sleeve gastroplasty; Laparoscopic sleeve gastroplasty; Obesity; Safety

\section{INTRODUCTION}

Obesity-associated metabolic conditions such as hypertension, type 2 diabetes, and dyslipidemia are significant health problems worldwide, and their prevalence rates are increasing. The most effective method for controlling obesity is bariatric surgery, which is more effective than diet and exercise or pharmacologic approaches. ${ }^{2}$ Laparoscopic sleeve gastrectomy

Received: December 5, 2020 Revised: January 3, 2021

Accepted: January 5, 2021

Correspondence: Jin Young Yoon

Department of Internal Medicine, Kyung Hee University Hospital, Kyung Hee University School of Medicine, 892 Dongnam-ro, Gandong-gu, Seoul 05278, Korea Tel: +82-2-440-6095, Fax: +82-2-440-6295, E-mail: htherehthere@gmail.com ORCID: https://orcid.org/0000-0002-5280-0443

It is the invited review article.

(c) This is an Open Access article distributed under the terms of the Creative Commons Attribution Non-Commercial License (http://creativecommons.org/ licenses/by-nc/3.0) which permits unrestricted non-commercial use, distribution, and reproduction in any medium, provided the original work is properly cited.
(LSG), a kind of bariatric surgery, involves resection of the greater curvature and fundus of the stomach under vertically oriented partial gastrectomy, resulting in reduction of the gastric volume by $75 \%-80 \%$ (Fig. $1 \mathrm{~A}$ ). ${ }^{3}$ Although LSG is usually associated with a high rate of weight loss, less than $1 \%$ of those who qualify for bariatric surgery actually undergo it because of the high cost and procedure-related complications. ${ }^{4}$

The endoscopic approach is a promising alternative modality for the treatment of obesity given its low financial burden and ease of accessibility requiring no incision. Currently, endoscopic methods and techniques are categorized into six class$e^{5}$ : (1) space-occupying devices such as intragastric balloons, (2) restrictive procedures such as endoscopic gastroplasty, (3) bypass liners, (4) electrical stimulation, (5) aspiration therapy, and (6) other therapies such as botulinum toxin A injection or duodenal mucosal resurfacing. Of these, restrictive procedures have been proven to be the most effective method for weight loss at 12 months. ${ }^{6}$

Endoscopic sleeve gastroplasty (ESG), a type of restrictive 


\section{C clinical enooscopy}

(A)
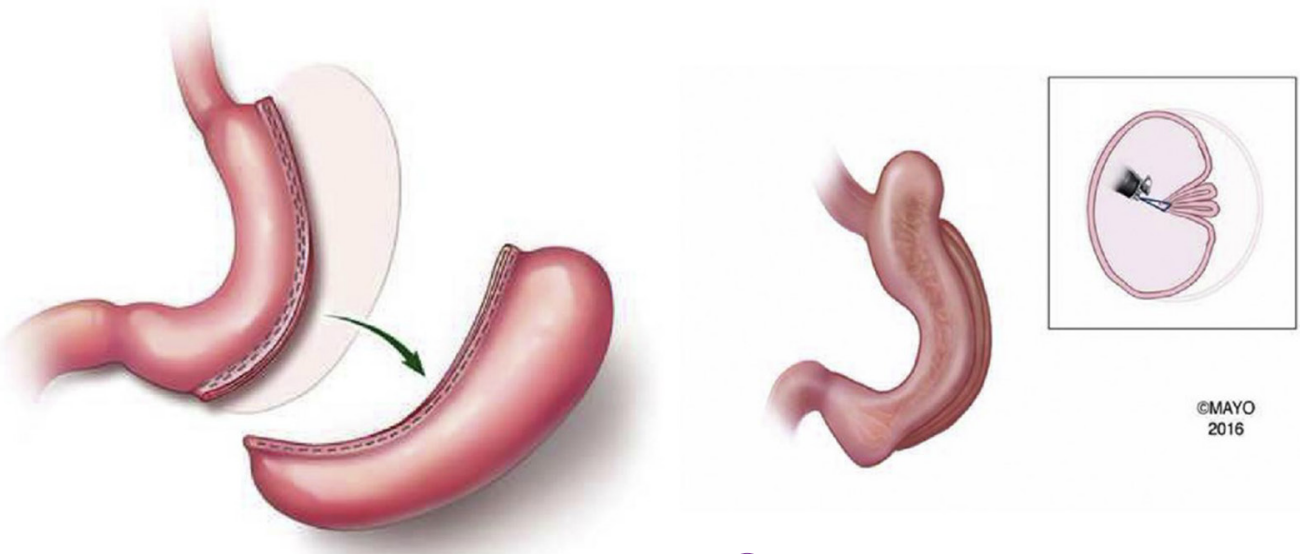

GMAYO
2016

(B)

Fig. 1. (A) Laparoscopic sleeve gastrectomy. (B) Endoscopic sleeve gastroplasty (used with permission of Mayo Foundation for Medical Education and Research, all rights reserved).

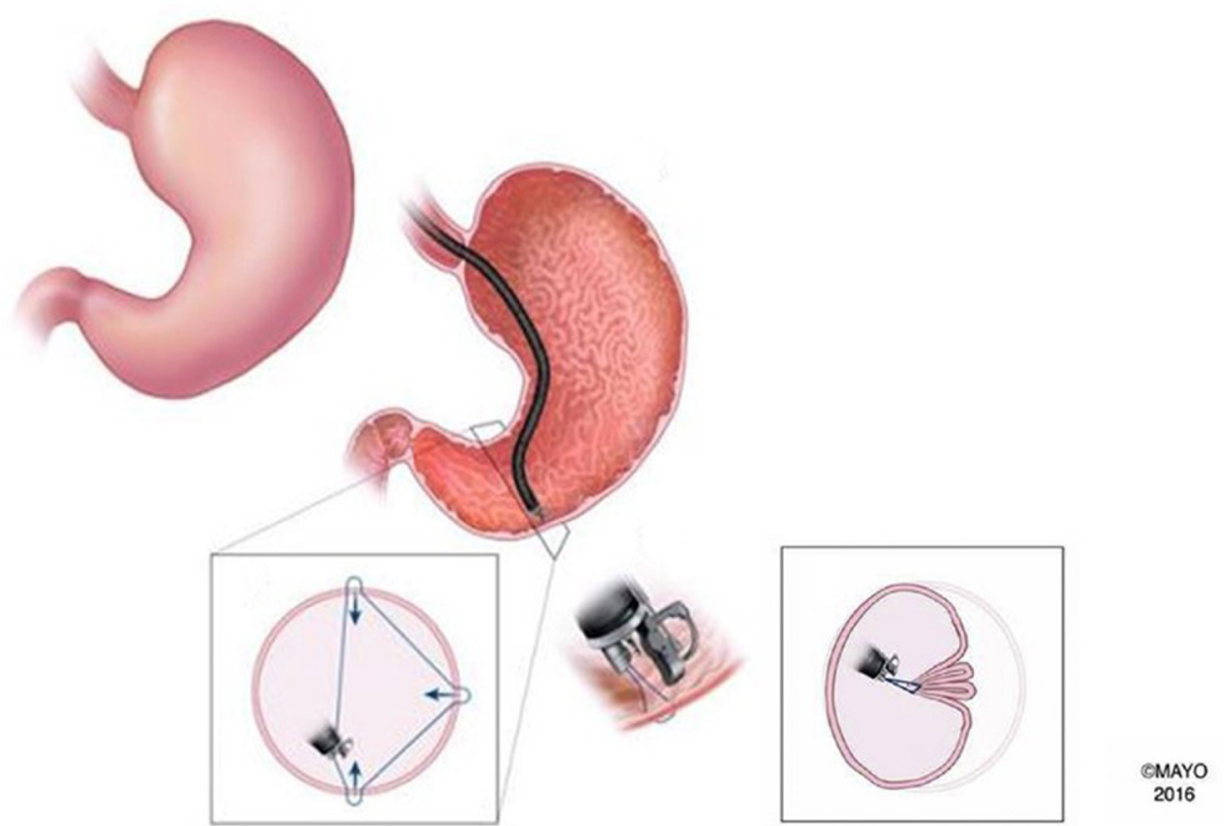

Fig. 2. Endoscopic sleeve gastroplasty performed using a full-thickness endoscopic suturing device (used with permission of Mayo Foundation for Medical Education and Research, all rights reserved).

procedure, is a novel therapeutic endoscopic technique first introduced by Abu Dayyeh et al. in 2013 (Fig. 1B). ${ }^{7}$ It is a minimally invasive technique that reduces the size of the gastric reservoir by using a full-thickness endoscopic suturing device, such as OverStitch ${ }^{\text {TM }}$ (Apollo Endosurgery, Austin, TX, USA). ${ }^{8}$
The anterior and posterior walls of the stomach are stitched together to achieve a tubular structure, similar to the structure achieved by LSG (Fig. 2). In this review article, we aimed to determine the effectiveness and safety profile of ESG and its usefulness as an alternative treatment for LSG. 


\section{EFFECTIVENESS OF ENDOSCOPIC SLEEVE GASTROPLASTY}

Cheskin et al. conducted a case-matched study that directly compared ESG with a combination of low-calorie diet, increased physical activity, and behavioral therapy, namely high-intensity diet and lifestyle therapy (HIDLT) as the firstline treatment for obesity. They examined 105 patients who underwent ESG and 281 patients who underwent HIDLT for comparing the efficacy of the interventions between the two groups. ${ }^{9}$ The ESG group had a significantly greater mean percentage of total body weight loss (\%TBWL) than the HIDLT group at 3 months ( $14.0 \%$ vs. $11.3 \%$, respectively) and at 12 months follow-up ( $20.6 \%$ vs. $14.3 \%$, respectively). Thus, ESG is a valuable alternative for patients with poor compliance to HIDLT.

Given the accumulated knowledge on the effectiveness of ESG in current studies, several systematic reviews and meta-analyses have been published; five recent systematic reviews and meta-analyses evaluated the effectiveness and safety of ESG for the treatment of obesity in the same year. ${ }^{10-14}$ First, Hedjoudje et al. included 1,772 patients from eight studies published between 2016 and 2019 and reported a 6-month mean $\%$ TBWL of $15.1 \%$, mean percentage of excess body weight loss (\%EBWL) of 57.7\%, and mean reduction in body mass index (BMI) of $5.65 \mathrm{~kg} / \mathrm{m}^{2} .{ }^{10}$ Weight loss was sustained at 1 year and at $18-24$ months with \%TBWL of $16.5 \%$ and $17.2 \%$, respectively. Second, in a meta-analysis of 2,170 patients from 11 studies published before October 2019, the pooled mean $\%$ TBWL values observed at 6,12 , and 18 months were $15.3 \%$, $16.1 \%$, and $16.8 \%$, respectively. The pooled mean $\% \mathrm{EBWL}$ values at 6,12 , and 18 months were $55.8 \%, 60 \%$, and $73 \%$, respectively. ${ }^{11}$ Third, Li et al. enrolled a total of 1,542 patients from nine studies published up to February 2019 and reported the pooled $\% \mathrm{TBWL}$ values of $8.8 \%, 11.9 \%, 14.5 \%$, and $16.1 \%$, respectively, at $1,3,6$, and 12 months. ${ }^{13}$ The pooled \%EBWL values at $1,3,6$, and 12 months were $31.2 \%, 43.6 \%, 53.1 \%$, and $59.1 \%$, respectively. ${ }^{13}$ Fourth, in a meta-analysis by Singh et al. with 1,859 patients from eight studies published before June 2019 , the pooled mean \%TBWL values at 6,12 , and 24 months were $14.9 \%, 16.4 \%$, and $20.0 \%$, respectively. ${ }^{14}$ The pooled mean \%EBWL values at 6,12 , and 24 months were $55.8 \%, 61.8 \%$, and $60.4 \%$, respectively. ${ }^{14}$ Lastly, Due-Petersson et al. included a total of 2,142 patients from 23 studies regardless of publication date and reported a $\% \mathrm{TBWL}$ of $16.3 \%$ at 12 months. ${ }^{12}$ In addition, ESG led to a significantly greater \%TBWL than intragastric balloon insertion ( $20.6 \%$ vs. $13.9 \%$ ) and HIDLT (20.6\% vs. $14.3 \%$ ), but significantly lower \%TBWL than LSG (17.1\% vs. $23.6 \%) .{ }^{12}$ Five recent systematic reviews and meta-analyses demonstrated that ESG was effective for weight loss in obese patients, with relatively narrow spectrum of weight loss ranging from $16.1 \%$ to $16.9 \%$ TBWL and $59.1 \%$ to $61.8 \%$ EBWL at 12 months of follow-up. This finding indicates that ESG is reproducible worldwide with effective weight loss outcomes. However, because none of the included studies were randomized controlled studies and most did not clarify adjuvant treatments such as nutritional care or pharmacotherapy during follow-up, these systematic reviews and meta-analyses did not show high-level evidence.

Neto et al. prospectively enrolled only patients with moderate obesity (Class I and Class II; BMI, 30-40 kg/m²) excluding those with severe obesity $\left(\mathrm{BMI}>40 \mathrm{~kg} / \mathrm{m}^{2}\right){ }^{15}$ The \%TBWL was $17.1 \%$ at 6 months and $19.7 \%$ at 12 months. Interestingly, the mean percentage of excess BMI loss was significantly greater among patients with Class I obesity than among those with Class II obesity at 6 (51.1\% vs. $43.9 \%)$ and 12 months (60.2\% vs. $49.2 \%$ ). These results suggest that ESG is a safe and effective option for patients with mild to moderate obesity as well as those with severe.

Most previous studies have reported clinical outcomes of ESG for up to 2 years after the procedure. Long-term studies on its effectiveness are lacking. Recently, Sharaiha et al. conducted a 5 -year analysis of a prospectively maintained cohort. ${ }^{16}$ At 1,3 , and 5 years, the mean $\% \mathrm{TBWL}$ values were $15.6 \%, 14.9 \%$, and $15.9 \%$, respectively. Further, $5 \%$ and $10 \%$ TBWL was maintained by $89 \%$ and $77 \%$ of the patients at 1 year, $85 \%$ and $63 \%$, at 3 years, and $90 \%$ and $61 \%$, at 5 years, respectively. These results suggest that ESG is effective for up to 5 years after the procedure, and its long-term durability is good for maintaining weight loss.

\section{COMPARISON OF ENDOSCOPIC SLEEVE GASTROPLASTY AND LAPAROSCOPIC SLEEVE GASTRECTOMY}

Three observational studies ${ }^{17-19}$ and two meta-analyses ${ }^{20,21}$ directly compared the effectiveness of ESG and LSG. An unmatched cohort study by Novikov et al. in 2018 reported that the \%TBWL with LSG was significantly superior at 12 months than that with ESG $(29.3 \%$ vs. $17.6 \%, p<0.001) .{ }^{17}$ This difference remained significant in patients with BMI $>40 \mathrm{~kg} /$ $\mathrm{m}^{2}$, but not in those with BMI $<40 \mathrm{~kg} / \mathrm{m}^{2}$. In a case-matched study conducted by Fayad et al. in 2019, there was a significantly greater \%TBWL in the ESG group than in the LSG group at the 1 -month follow-up ( $9.8 \%$ vs. $6.6 \%, p<0.001)$, whereas it was lower in the ESG group than in the LSG group at 6 months $(17.1 \%$ vs. $23.6 \%, p<0.001) .{ }^{18}$ Similar to an earlier 
study, \%TBWL remained significantly low in the ESG group in patients with BMI $>40 \mathrm{~kg} / \mathrm{m}^{2}$, with a borderline significant difference in those with BMI $<40 \mathrm{~kg} / \mathrm{m}^{2}$. Both studies showed that weight loss with the endoscopic and surgical approaches was equivalent in patients with BMI $<40 \mathrm{~kg} / \mathrm{m}^{2}$. However, as the BMI increases, bariatric surgery is likely to be more effective than ESG. These findings indicate that the endoscopic approach could be preferentially considered in patients with BMI between 30 and $40 \mathrm{~kg} / \mathrm{m}^{2}$, although those with BMI $>40$ $\mathrm{kg} / \mathrm{m}^{2}$ should be assessed for bariatric surgery, considering the extensive data supporting weight loss with the surgical option. A recent study compared ESG and LSG with 135 ESG and 43 LSG patients followed for 1 year and 46 ESG and 34 LSG patients followed for 2 years ${ }^{19}$; this study showed that the mean \%TBWL values at 2 years for ESG and LSG were 18.5\% and $28.3 \%$, respectively $(p<0.001)$. Both procedures achieved maximum weight loss in the 18 months, with gradual regression starting from 12 months.

Among two meta-analysis comparing ESG with LSG, one included 1,451 ESG and 203 LSG patients from five studies and found pooled \% TBWL values of $14.2 \%, 15.2 \%, 14.8 \%$, and $18.6 \%$ at $6,12,18$, and 24 months, respectively, with ESG. ${ }^{20}$ Meanwhile, LSG showed high pooled \%TBWL values of $23.5 \%$ at 6 months and $29.3 \%$ at 12 months. ${ }^{20}$ A meta-analysis of two studies directly comparing LSG and ESG showed significant differences in the mean \%TBWL (8.52; 95\% CI, $6.35-10.69 ; p<0.00001)$ at 6 months, favoring LSG over ESG. ${ }^{17,18,20}$ In another meta-analysis of eight studies with 1,815 ESG patients and seven studies with 2,179 LSG patients, the pooled rates of \%TBWL with LSG were statistically superior at 12 months to the rates with ESG ( $30.5 \%$ vs. $17.1 \%, p=0.001){ }^{21}$ Current evidence indicates that ESG offers satisfactory efficacy in patients with mild-to moderate and inferior efficacy to LSG in those with severe obesity; it is better than other endoscopic procedures like intra-gastric balloon. ${ }^{22}$

\section{PREDICTIVE FACTORS FOR HIGH WEIGHT LOSS}

Not all patients respond well to ESG, and identifying predictors for patient response is an important aspect in subject selection and peri-procedural care. Technical factors such as the pattern and number of sutures or intervals in the stomach length have not been found to be independent predictors of weight loss. ${ }^{16,23}$

A recent study found that young age, high TBWL at 1 month after ESG, compliance with scheduled visit, and endoscopist's experience, in terms of number of cases, were predic- tors of high TBWL at the follow-up after ESG ${ }^{16}$ Another study found that high TBWL was correlated with young age and endoscopists with experience of $>35$ cases. ${ }^{24} \mathrm{~A}$ recent study on the Indian population showed that age of less than 30 years and female sex were related to increased weight loss after ESG at 12 months. ${ }^{25}$ Meanwhile, another study reported that male sex was a significant predictive factor for achieving $>10 \%$ TBWL at 6 months, but not at 12 months. ${ }^{26}$

A previous study on the learning curve for ESG reported that efficiency and mastery in ESG was reached after 38 and 55 procedures, respectively. ${ }^{27}$ Meanwhile, LSG learning curves have been reported to be as low as 28 cases. ${ }^{28}$ Endoscopist's experience as a predictive factor means that standardization of ESG training programs is needed to ensure reproducible efficacy and safety. A detailed study on the learning curve of ESG found that endoscopists who wanted to reduce procedure time and number of plications per procedure could achieve progress plateau at 7 and 9 successive cases, respectively. ${ }^{29} \mathrm{~A}$ study on the first Indian experience showed that 35 cases were required to achieve reduction in procedure time and effective weight loss and safety profile. ${ }^{25}$

Lopez-Nava et al. confirmed that constant monitoring for managing patients is a significant issue. ${ }^{19}$ They defined completers as those who reached 2 years of follow-up and non-completers as those who dropped out after the first year and found that completers achieved significantly higher mean \%TBWL than non-completers both with ESG (20.5\% vs. $16.9 \%)$ and LSG (30.1\% vs. $26.5 \%)$ at 1 year. Another study also supported that adherence to multidisciplinary team follow-up was an independent factor for increased weight loss at 1 year, but not in type of endoscopic procedures. ${ }^{30}$ Provision of opportunity for nutritional and psychological consultation was also predictive of successful weight loss. ${ }^{31}$ These results emphasized the importance of regular monitoring by experts in various fields after the procedure.

\section{MECHANISMS OF WEIGHT LOSS INDUCED BY ENDOSCOPIC SLEEVE GASTROPLASTY}

The changes in anatomical configuration induced by ESG might be an essential element of weight loss, but the exact mechanism of weight loss after the procedure is debatable. The proposed mechanisms include delayed gastric emptying, increased early satiation, and possibly alteration of the gut and metabolic hormones. ${ }^{32}$

Although ESG is structurally analogous to LSG, the mechanisms of weight loss are different between these procedures 
in terms of gut and metabolic hormones. Lopez-Nava et al. found that in patients after ESG, leptin and insulin levels decreased with improvement in insulin secretion patterns and no changes in fasting ghrelin, glucagon-like peptide (GLP-1), and peptide-YY (PYY) levels at 6 months. ${ }^{33}$ Meanwhile, after LSG, patients showed a significant increase in GLP-1, PYY, and adiponectin levels and a decrease in ghrelin and leptin levels at 6 months. The observed differences in these hormone changes are likely associated with the anatomical differences between the two procedures. In ESG, the gastric fundus, which acts as a reservoir to store food, and neuronal innervation are left intact, and consequently stasis and delayed transit of food stimulates early satiety through the signals origination from the stomach to the brain. ${ }^{34}$ On the other hand, in LSG, the excised gastric fundus and up to $80 \%$ reduction of gastric volume with disconnection of the gastric nerves promote early emptying of food contents into the small bowel, and consequently, various gut and metabolic hormones undergo unusual alterations. ${ }^{33}$

These explanations were supported by several studies that measured emptying time. Vargas et al. showed that LSG reduced gastric emptying $\mathrm{T}^{1} / 2$ by 29.2 minutes and accelerated emptying of solid gastric contents in particular. ${ }^{35}$ In contrast, ESG increased gastric emptying T112 by 90 minutes and delayed gastric emptying for solids. ${ }^{36}$ The retention of food after ESG led to early meal termination in 11 minutes and reduced food intake. ${ }^{32}$ Thus, gut hormone changes play a minor role in weight loss after ESG and changes in gastric emptying and time to satiation are some of the plausible mechanisms that lead to beneficial effects of ESG.

\section{SAFETY OF ENDOSCOPIC SLEEVE GASTROPLASTY}

We speculated that the minimally invasive ESG procedure is relatively safe, as per current evidence. Mild adverse events (AEs) such as abdominal pain, nausea, and vomiting occurred immediately after the procedure, but almost all of the symptoms improved after a few days with conservative management or spontaneously regressed. ${ }^{13}$

There is a risk of ESG-related infection. The full-thickness sutures performed during ESG may lead to intraperitoneal contamination by gastric contents and bacterial translocation. Thus, antibiotic prophylaxis with cefazolin ( 1 to $2 \mathrm{~g}$ ) is recommended an hour before the procedure. ${ }^{37}$ Tissue tearing around the suture site by excessive tension can cause large perforations as well as microperforations, resulting in perigastric fluid collection or abscess formation. Most of the fluid collections identified on computed tomography were resolved by antibi- otic administration alone, with occasional radiologic intervention, and rarely required surgical procedure. AEs associated with gastric leak were reported in $<1 \%$ of cases. ${ }^{10}$

A meta-analysis of 1,772 patients reported a pooled rate of severe AEs of 2.2\% after ESG. ${ }^{10}$ The reported AEs were pain or nausea requiring hospitalization in 18 cases (1.08\%), upper gastrointestinal (GI) bleeding in nine cases $(0.56 \%)$, perigastric leak or collection in eight cases $(0.48 \%)$, pulmonary embolism in one case $(0.06 \%)$, and pneumoperitoneum in one case $(0.06 \%))^{10}$ Another recent systematic review and meta-analysis including 2,170 patients from 11 studies observed an overall rate of AEs of $2.3 \%$, consisting of $1.5 \%$ of mild, $1.7 \%$ of moderate, and $0.8 \%$ of severe $\mathrm{AEs}^{11}$; this study reported a total of 38 AEs including 13 cases of GI bleeding, 10 cases of perigastric fluid collection, eight cases of severe abdominal pain, five cases of fever, one case of deep vein thrombosis treated with full anticoagulation, and one case of pneumothorax requiring thoracic drainage. ${ }^{11}$ Most of major AEs were managed conservatively, except two cases of GI bleeding requiring sclerotherapy and three cases of perigastric fluid collection requiring surgical drainage and closure. A study by Li et al. reported that the pooled rate of mild AEs such as self-limited abdominal pain and nausea was $72 \%$, and that of severe adverse events (SAEs) such as perigastric fluid collection, GI bleeding, pulmonary embolism, pneumoperitoneum, and pneumothorax was only $1 \%{ }^{13}$ The pooled incidence of SAEs was $2.3 \%$ in the meta-analysis by Singh et al. and 1.5\% the study by Due-Petersson et al. ${ }^{12,14}$ No procedure-related mortality was reported in any of the included studies. Several recent meta-analyses confirmed that ESG could be introduced as a safe clinical practice.

ESG is generally associated with significantly lower AEs than LSG. Three observational studies showed significant differences in the AEs between ESG and LSG, i.e., 5.2\% vs. 16.9\% in the study by Fayad et al., 2.2\% vs. 9.2\% in the study by Novikov et al., and $0.5 \%$ vs. $4.9 \%$ in the study by Lopez-Nava et al. ${ }^{17-19}$ In a meta-analysis study, the pooled rate of all AEs with ESG was $2.9 \%$ and with LSG was $11.8 \%(p=0.001) .{ }^{21}$ ESG had a significantly lower incidence of bleeding events (1.1\% vs. $2.6 \%, p=0.005)$ and gastroesophageal reflux disease (GERD) $(0.4 \%$ vs. $5.8 \%, p=0.001)$ than $\mathrm{LSG}^{21}$ Several recent meta-analyses demonstrated that ESG has a better safety profile than LSG.

GERD is known to be a considerable sequela to LSG. The development of GERD after LSG can be explained by the low resting esophageal sphincter pressure and low maximal distal contraction integral. ${ }^{38}$ The incidence rate of new-onset GERD after LSG was up to $34 \%$, as reported in a systematic review and up to $60 \%$ during a mean follow-up of 5.5 years after 
LSG. ${ }^{39,40}$ Fayad et al. found that the incidence of new-onset GERD was significantly lower after ESG than after LSG (1.9\% vs. $14.5 \%) .{ }^{18}$ This lower rate of GERD after ESG was explained by the fact that the fundus of the stomach is left intact and the neuronal innervation of the stomach is maintained. ${ }^{41}$ In a recent meta-analysis study of 1,772 patients from eight studies between 2016 and 2019, GERD was not listed as a SAE in any study. ${ }^{10}$ Therefore, the rate of new-onset GERD after ESG is negligible and may cause patients to see ESG as a more favorable treatment option than LSG.

In addition to the safety profile, the mean procedure time is shorter for ES than for LSG (45-80 min vs. $60-120 \mathrm{~min}$, respectively) so is the mean hospital length of stay (1-2 days vs. 5-9 days, respectively). ${ }^{21}$ It was noted that same-day discharge after LSG was associated with increased overall rates of morbidity, readmission, and reoperation. ${ }^{42}$ In contrast, no AEs have been during the initial in-hospital recovery period after ESG and all patients can be discharged on the same day. ${ }^{18}$

\section{ADJUVANT TREATMENT AFTER ENDOSCOPIC SLEEVE GASTROPLASTY}

A recent study on the effect of adjunct pharmacotherapy in patients undergoing ESG found that the combination of ESG and liraglutide was more effective than ESG alone. ${ }^{43}$ Liraglutide is a GLP-1 agonist that amplifies glucose-stimulated insulin secretion, delays gastric emptying, and increases satiety via the central effects on the hypothalamus. ${ }^{44}$ Pharmacotherapy with only liraglutide also showed favorable results of TBWL of $10 \%-15 \%{ }^{45}$ The combination group had significantly higher mean \%TBWL than the ESG alone group at 7 months $(24.7 \pm 2.1 \%$ vs. $20.5 \pm 1.7 \%, p<0.001)$ and also greater reduction in percent body fat at 12 months ( $7.9 \pm 1.3$ vs. $10.5 \pm 1.9$, $p<0.001)$. On the contrary, a study with 5 -year long-term outcomes of ESG revealed that post-procedural adjunct pharmacotherapy might not induce noticeably further weight loss but prevent further weight gain in patients. ${ }^{16}$ Clinicians should support that patients with obesity recognize the importance of multidisciplinary and comprehensive post-procedural care plans with respect to nutritional planning, endocrinology consultation, physical training, and even psychiatric care.

Furthermore, ESG is increasingly being used in salvage management for weight regain after LSG. ${ }^{46}$ In a study conducted by de Moura et al. with 34 patients who underwent ESG for weight regain after sleeve gastrectomy, the technical success rate was $100 \%$ with no SAEs and $\geq 25 \%$ EBWL in all patients, with a mean \%TBWL of $18.3 \%$ at 12 months. ${ }^{46}$ One case report on conversion to surgical treatment in patients with failed weight loss after ESG demonstrated to be feasible to remove suture and hardware relating to ESG and then perform safe stapling and pouch formation during bariatric surgery. ${ }^{47}$ The clinical outcomes and safety profile of revisional bariatric surgery require further examination.

\section{EFFECT OF ENDOSCOPIC SLEEVE GASTROPLASTY ON METABOLIC COMORBIDITY}

Achieving at least 10\% TBWL after obesity treatment improves obesity-related metabolic diseases. ${ }^{48}$ The mean \%TBWL of ESG has been reported to be more than $16 \%$, and it is considered beneficial in light of the threshold value. Several studies have demonstrated that endoscopic bariatric procedures such as ESG can reduce obesity-associated comorbidities. A study reported by Sharaiha et al. demonstrated that ESG significantly reduced $\mathrm{HbA1c}$ levels from $6.1 \%$ to $5.5 \%$, systolic blood pressure from $129 \mathrm{~mm} \mathrm{Hg}$ to $122 \mathrm{~mm} \mathrm{Hg}$, and triglyceride levels from $131.8 \mathrm{mmol} / \mathrm{dL}$ to $92.4 \mathrm{mmol} / \mathrm{dL}$ at 12 months. ${ }^{24}$ Alqahtani et al. reported complete remission of diabetes in $76.5 \%$ of the patients at 3 months and that of hypertension and dyslipidemia in $100 \%$ and $56.3 \%$ at 12 months, respectively. ${ }^{49}$ A recent study showed a significant decrease in the homeostatic model assessment of insulin resistance (HOMA-IR) and leptin level at 6 months. ${ }^{50}$ Several studies have shown the benefits of ESG in terms of hepatic problem. There was a reduction in serum alanine aminotransferase (ALT) level at 12 months from $42 \mathrm{IU} / \mathrm{L}$ to $22 \mathrm{IU} / \mathrm{L}$ in men and from $28 \mathrm{IU} / \mathrm{L}$ to $20 \mathrm{IU} / \mathrm{L}$ in women. ${ }^{24}$ ESG has been proven to be effective in patients with both obesity and non-alcoholic fatty liver disease (NAFLD) in terms of the risk of hepatic steatosis (NAFLD-fibrosis score), insulin resistance (HOMA-IR), and insulin and triglyceride levels at 12 months. ${ }^{51}$ These ameliorating effects of ESG on insulin resistance and changes in hepatic steatosis and fibrosis lasted for 2 years after the procedure. ${ }^{52}$

Obese patients have lower quality of life (QOL) than the healthy general population. ESG leads to not only significant weight loss but also improvement in health-related QOL and physical activity and is particularly beneficial for patients with high initial BMI and physical inactivity at baseline. ${ }^{53}$ In a study evaluating QOL measured by the Gastrointestinal Quality of Life Index with propensity-matching score analysis between ESG and LSG, it was worth noting that the ESG group, despite having significant low $\% \mathrm{EBWL}(39.9 \%$ vs. $54.9 \%, p=0.01)$ and $\%$ TBWL (13.4\% vs. $18.8 \%, p=0.03$ ) presented improved QOL with clear benefits in the GI symptom subdomain, while the LSG group showed a worsening of GERD symptoms (30.7\% 
vs. $0 \%$ ) and increased use of PPI therapy. ${ }^{54}$ While the overall weight loss after ESG is lower than that after LSG, ESG seems to be a good modality for preserving weight reduction and even improving QOL.

\section{CONCLUSIONS}

According to the minimal thresholds of 25\% EBWL and $<5 \%$ SAE recommended by the American Society for Gastrointestinal Endoscopy and the American Society for Metabolic and Bariatric Surgery joint task force for proving effective bariatric treatment, ESG with a reported mean SAE rate of $1.5 \%-2.3 \%$ and $\%$ EBWL of $59.1 \%-61.8 \%$ could qualify as a safe and primary endoscopic bariatric intervention. ${ }^{55}$

Considering the increasing evidence supporting the efficacy and safety of ESG, it appears to be more cost-effective and have a better safety profile than LSG. Hence, ESG can be considered as an option for the obese population, especially those with mild-to-moderate obesity. Thus, endoscopists are positioned to play a pivotal role in the future treatment of obesity. Unfortunately, in Korea, appropriate government-certified procedures covered by insurance are lacking, and this remains a significant barrier to the widespread use of endobariatric techniques. It is difficult to adequately compare the cost-effectiveness of ESG and LSG because LSG is covered by insurance for the eligible obese population, while ESG is mostly a selfpay procedure in Korea. In addition, the relatively high prevalence of gastric cancer in Korea makes endoscopists to hesitate performance of ESG inevitably remaining blind gastric mucosa.

In conclusion, ESG appears to be an effective alternative to LSG for obese patients who are not suitable for or unwilling to undergo a surgical procedure. Although LSG resulted in greater weight loss than ESG at 12 months, ESG has a better safety profile and shorter procedure time and length of hospital stay than LSG and is even reversible. Future studies and randomized controlled trials are needed to identify the longterm safety and efficacy of ESG and compare the advantages and cost-effectiveness against between ESG and LSG.

Conflicts of Interest

The authors have no potential conflicts of interest.

Funding
None.

Author Contributions

Conceptualization: Jin Young Yoon, The Study Group for Endoscopic Bariatric and Metabolic Therapies in the Korean Society of Gastrointestinal Endoscopy
Writing-original draft: JYY

Writing-review\&editing: Román Turró Arau

ORCID

Jin Young Yoon:

Román Turró Arau:

https://orcid.org/0000-0002-5280-0443 https://orcid.org/0000-0002-3433-8689

\section{REFERENCES}

1. Rønningen R, Wammer ACP, Grabner NH, Valderhaug TG. Associations between lifetime adversity and obesity treatment in patients with morbid obesity. Obes Facts 2019;12:1-13.

2. Gloy VL, Briel M, Bhatt DL, et al. Bariatric surgery versus non-surgical treatment for obesity: a systematic review and meta-analysis of randomised controlled trials. BMJ 2013;347:f5934.

3. Ali M, El Chaar M, Ghiassi S, Rogers AM. American Society for Metabolic and Bariatric Surgery updated position statement on sleeve gastrectomy as a bariatric procedure. Surg Obes Relat Dis 2017;13:16521657.

4. Chang SH, Stoll CR, Song J, Varela JE, Eagon CJ, Colditz GA. The effectiveness and risks of bariatric surgery: an updated systematic review and meta-analysis, 2003-2012. JAMA Surg 2014;149:275-287.

5. ASGE Bariatric Endoscopy Task Force; ASGE Technology Committee; Abu Dayyeh BK, et al. Endoscopic bariatric therapies. Gastrointest Endosc 2015;81:1073-1086.

6. Neylan CJ, Dempsey DT, Tewksbury CM, Williams NN, Dumon KR. Endoscopic treatments of obesity: a comprehensive review. Surg Obes Relat Dis 2016;12:1108-1115.

7. Abu Dayyeh BK, Rajan E, Gostout CJ. Endoscopic sleeve gastroplasty: a potential endoscopic alternative to surgical sleeve gastrectomy for treatment of obesity. Gastrointest Endosc 2013;78:530-535.

8. Lopez-Nava G, Galvão MP, Bautista-Castaño I, Jimenez-Baños A, Fernandez-Corbelle JP. Endoscopic sleeve gastroplasty: how I do it? Obes Surg 2015;25:1534-1538.

9. Cheskin LJ, Hill C, Adam A, et al. Endoscopic sleeve gastroplasty versus high-intensity diet and lifestyle therapy: a case-matched study. Gastrointest Endosc 2020;91:342-349.e1.

10. Hedjoudje A, Abu Dayyeh BK, Cheskin LJ, et al. Efficacy and safety of endoscopic sleeve gastroplasty: a systematic review and meta-analysis. Clin Gastroenterol Hepatol 2020;18:1043-1053.e4.

11. de Miranda Neto AA, de Moura DTH, Ribeiro IB, et al. Efficacy and safety of endoscopic sleeve gastroplasty at mid term in the management of overweight and obese patients: a systematic review and meta-analysis. Obes Surg 2020;30:1971-1987.

12. Due-Petersson R, Poulsen IM, Hedbäck N, Karstensen JG. Effect and safety of endoscopic sleeve gastroplasty for treating obesity - a systematic review. Dan Med J 2020;67:A05200359.

13. Li P, Ma B, Gong S, Zhang X, Li W. Efficacy and safety of endoscopic sleeve gastroplasty for obesity patients: a meta-analysis. Surg Endosc 2020;34:1253-1260.

14. Singh S, Hourneaux de Moura DT, Khan A, Bilal M, Ryan MB, Thompson CC. Safety and efficacy of endoscopic sleeve gastroplasty worldwide for treatment of obesity: a systematic review and meta-analysis. Surg Obes Relat Dis 2020;16:340-351.

15. Neto MG, Moon RC, de Quadros LG, et al. Safety and short-term effectiveness of endoscopic sleeve gastroplasty using overstitch: preliminary report from a multicenter study. Surg Endosc 2020;34:4388-4394.

16. Sharaiha RZ, Hajifathalian K, Kumar R, et al. Five-year outcomes of endoscopic sleeve gastroplasty for the treatment of obesity. Clin Gastroenterol Hepatol 2020 Oct 1 [Epub]. https://doi.org/10.1016/ j.cgh.2020.09.055.

17. Novikov AA, Afaneh C, Saumoy M, et al. Endoscopic sleeve gastroplas- 
ty, laparoscopic sleeve gastrectomy, and laparoscopic band for weight loss: how do they compare? J Gastrointest Surg 2018;22:267-273.

18. Fayad L, Adam A, Schweitzer M, et al. Endoscopic sleeve gastroplasty versus laparoscopic sleeve gastrectomy: a case-matched study. Gastrointest Endosc 2019;89:782-788.

19. Lopez-Nava G, Asokkumar R, Bautista-Castaño I, et al. Endoscopic sleeve gastroplasty, laparoscopic sleeve gastrectomy, and laparoscopic greater curve plication: do they differ at 2 years? Endoscopy $2020 \mathrm{Jul} 22$ [Epub]. https://doi.org/10.1055/a-1224-7231.

20. Jalal MA, Cheng Q, Edye MB. Systematic review and meta-analysis of endoscopic sleeve gastroplasty with comparison to laparoscopic sleeve gastrectomy. Obes Surg 2020;30:2754-2762.

21. Mohan BP, Asokkumar R, Khan SR, et al. Outcomes of endoscopic sleeve gastroplasty; how does it compare to laparoscopic sleeve gastrectomy? A systematic review and meta-analysis. Endosc Int Open 2020;8:E558-E565

22. Singh S, de Moura DTH, Khan A, et al. Intragastric balloon versus endoscopic sleeve gastroplasty for the treatment of obesity: a systematic review and meta-analysis. Obes Surg 2020;30:3010-3029.

23. Espinet-Coll E, Nebreda-Durán J, Galvao-Neto M, et al. Suture pattern does not influence outcomes of endoscopic sleeve gastroplasty in obese patients. Endosc Int Open 2020;8:E1349-E1358.

24. Sharaiha RZ, Kumta NA, Saumoy M, et al. Endoscopic sleeve gastroplasty significantly reduces body mass index and metabolic complications in obese patients. Clin Gastroenterol Hepatol 2017;15:504-510.

25. Bhandari M, Jain S, Mathur W, et al. Endoscopic sleeve gastroplasty is an effective and safe minimally invasive approach for treatment of obesity: first Indian experience. Dig Endosc 2020;32:541-546.

26. Barrichello S, Hourneaux de Moura DT, Hourneaux de Moura EG, et al. Endoscopic sleeve gastroplasty in the management of overweight and obesity: an international multicenter study. Gastrointest Endosc 2019;90:770-780.

27. Saumoy M, Schneider Y, Zhou XK, et al. A single-operator learning curve analysis for the endoscopic sleeve gastroplasty. Gastrointest Endosc 2018;87:442-447.

28. Prevot F, Verhaeghe P, Pequignot A, et al. Two lessons from a 5-year follow-up study of laparoscopic sleeve gastrectomy: persistent, relevant weight loss and a short surgical learning curve. Surgery 2014;155:292299.

29. Hill C, El Zein M, Agnihotri A, et al. Endoscopic sleeve gastroplasty: the learning curve. Endosc Int Open 2017;5:E900-E904.

30. Lopez-Nava G, Asokkumar R, Rull A, Corbelle F, Beltran L, Bautista I. Bariatric endoscopy procedure type or follow-up: what predicted success at 1 year in 962 obese patients? Endosc Int Open 2019;7:E1691-E1698.

31. Lopez-Nava G, Galvao M, Bautista-Castaño I, Fernandez-Corbelle JP, Trell M. Endoscopic sleeve gastroplasty with 1-year follow-up: factors predictive of success. Endosc Int Open 2016;4:E222-E227.

32. Abu Dayyeh BK, Acosta A, Camilleri M, et al. Endoscopic sleeve gastroplasty alters gastric physiology and induces loss of body weight in obese individuals. Clin Gastroenterol Hepatol 2017;15:37-43.e1.

33. Lopez-Nava G, Negi A, Bautista-Castaño I, Rubio MA, Asokkumar R. Gut and metabolic hormones changes after endoscopic sleeve gastroplasty (ESG) vs. laparoscopic sleeve gastrectomy (LSG). Obes Surg 2020;30:2642-2651.

34. Jirapinyo P, Thompson CC. Endoscopic bariatric and metabolic therapies: surgical analogues and mechanisms of action. Clin Gastroenterol Hepatol 2017;15:619-630.

35. Vargas EJ, Bazerbachi F, Calderon G, et al. Changes in time of gastric emptying after surgical and endoscopic bariatrics and weight loss: a systematic review and meta-analysis. Clin Gastroenterol Hepatol 2020;18:57-68.e5.

36. Abu Dayyeh B, Rizk M, El-Mohsen MA, et al. Laparoscopic greater curvature plication (LGCP) vs. endoscopic sleeve gastroplasty (ESG): similar efficacy with different physiology. Surg Obes Relat Dis 2017;13(10 Suppl):S205.
37. de Moura DTH, Badurdeen DS, Ribeiro IB, Leite E, Thompson CC, Kumbhari V. Perspectives toward minimizing the adverse events of endoscopic sleeve gastroplasty. Gastrointest Endosc 2020;92:1115-1121.

38. Quero G, Fiorillo C, Dallemagne B, et al. The causes of gastroesophageal reflux after laparoscopic sleeve gastrectomy: quantitative assessment of the structure and function of the esophagogastric junction by magnetic resonance imaging and high-resolution manometry. Obes Surg 2020;30:2108-2117.

39. Oor JE, Roks DJ, Ünlü Ç, Hazebroek EJ. Laparoscopic sleeve gastrectomy and gastroesophageal reflux disease: a systematic review and meta-analysis. Am J Surg 2016;211:250-267.

40. Soricelli E, Casella G, Baglio G, Maselli R, Ernesti I, Genco A. Lack of correlation between gastroesophageal reflux disease symptoms and esophageal lesions after sleeve gastrectomy. Surg Obes Relat Dis 2018;14:751-756.

41. Asokkumar R, Babu MP, Bautista I, Lopez-Nava G. The use of the OverStitch for bariatric weight loss in Europe. Gastrointest Endosc Clin N Am 2020;30:129-145.

42. Inaba CS, Koh CY, Sujatha-Bhaskar S, Pejcinovska M, Nguyen NT. How safe is same-day discharge after laparoscopic sleeve gastrectomy? Surg Obes Relat Dis 2018;14:1448-1453.

43. Badurdeen D, Hoff AC, Hedjoudje A, et al. Endoscopic sleeve gastroplasty plus liraglutide versus endoscopic sleeve gastroplasty alone for weight loss. Gastrointest Endosc 2020 Oct 17 [Epub]. https://10.1016/ j.gie.2020.10.016.

44. Nauck MA, Niedereichholz U, Ettler R, et al. Glucagon-like peptide 1 inhibition of gastric emptying outweighs its insulinotropic effects in healthy humans. Am J Physiol 1997;273:E981-E988.

45. Nuffer WA, Trujillo JM. Liraglutide: a new option for the treatment of obesity. Pharmacotherapy 2015;35:926-934.

46. de Moura DTH, Barrichello S, Jr., de Moura EGH, et al. Endoscopic sleeve gastroplasty in the management of weight regain after sleeve gastrectomy. Endoscopy 2020;52:202-210.

47. Beitner M, Hopkins G. Conversion of endoscopic sleeve gastroplasty to laparoscopic Roux-en-Y gastric bypass. Surg Obes Relat Dis 2020;16:590-591.

48. Vilar-Gomez E, Martinez-Perez Y, Calzadilla-Bertot L, et al. Weight loss through lifestyle modification significantly reduces features of nonalcoholic steatohepatitis. Gastroenterology 2015;149:367-378.e5; quiz e14-e15.

49. Alqahtani A, Al-Darwish A, Mahmoud AE, Alqahtani YA, Elahmedi M. Short-term outcomes of endoscopic sleeve gastroplasty in 1000 consecutive patients. Gastrointest Endosc 2019;89:1132-1138.

50. Mehta A, Hajifathalian K, Dawod QM, et al. An analysis of leptin and insulin-glucose metabolism following endoscopic sleeve gastroplasty. Gastroenterology 2019;156(6 Suppl 1):S-236.

51. Espinet Coll E, Vila Lolo C, Díaz Galán P, et al. Bariatric and metabolic endoscopy in the handling of fatty liver disease. A new emerging approach? Rev Esp Enferm Dig 2019;111:283-293.

52. Hajifathalian K, Mehta A, Ang B, et al. Improvement in insulin resistance and estimated hepatic steatosis and fibrosis after endoscopic sleeve gastroplasty. Gastrointest Endosc 2020 Aug 27 [Epub]. https://10.1016/ j.gie.2020.08.023.

53. Fiorillo C, Quero G, Vix M, et al. 6-month gastrointestinal quality of life (QoL) results after endoscopic sleeve gastroplasty and laparoscopic sleeve gastrectomy: a propensity score analysis. Obes Surg 2020;30:19441951.

54. Lopez-Nava G, Asokkumar R, Lacruz T, Rull A, Beltran L, Bautista-Castaño I. The effect of weight loss and exercise on health-related quality of life (HRQOL) following endoscopic bariatric therapies (EBT) for obesity. Health Qual Life Outcomes 2020;18:130.

55. Abu Dayyeh BK, Kumar N, Edmundowicz SA, et al. ASGE Bariatric Endoscopy Task Force systematic review and meta-analysis assessing the ASGE PIVI thresholds for adopting endoscopic bariatric therapies. Gastrointest Endosc 2015;82:425-438.e5. 